\title{
Curcumin ameliorates ischemic stroke injury in rats by protecting the integrity of the blood-brain barrier
}

\author{
SHUGUANG WU ${ }^{1 *}$, TING GUO ${ }^{2 *}$, WENXUAN QI ${ }^{3}$, YUYU LI $^{3}, \mathrm{JIE} \mathrm{GU}^{3}, \mathrm{CUI} \mathrm{LIU}^{3}$, \\ YUEHONG SHA ${ }^{4}$, BAOCHENG YANG ${ }^{3,5}$, SHUQUN HU ${ }^{3,5}$ and XUEMEI ZONG ${ }^{3,5}$ \\ ${ }^{1}$ Department of Anesthesiology, The Second Affiliated Hospital of Zhejiang University School of Medicine, Hangzhou, \\ Zhejiang 313000; ${ }^{2}$ Department of Neurology, Fujian Medical University Union Hospital, Fuzhou, Fujian 350001; \\ ${ }^{3}$ Jiangsu Provincial Institute of Health Emergency, Xuzhou Medical University, Xuzhou, Jiangsu 221002; \\ ${ }^{4}$ Department of Emergency, First People's Hospital, Pizhou, Jiangsu 221300; ${ }^{5}$ Emergency Center of \\ The Affiliated Hospital of Xuzhou Medical University, Xuzhou, Jiangsu 221002, P.R. China
}

Received September 30, 2020; Accepted April 16, 2021

DOI: $10.3892 / \mathrm{etm} .2021 .10215$

\begin{abstract}
The blood-brain barrier (BBB) is critical for proper cerebral homeostasis and its dysfunction during ischemic stroke can result in significant neurological injury. The major goal of the present study was to identify whether curcumin pretreatment possessed protective effects on BBB integrity during the $24 \mathrm{~h}$ of acute ischemic brain injury. To investigate the protective effects of curcumin, male Sprague-Dawley rats were divided into multiple groups, including sham, middle cerebral artery occlusion/reperfusion (MCAO/R) vehicle and curcumin pretreated $\mathrm{MCAO} / \mathrm{R}$ groups. The effects of curcumin were measured by analyzing neurological deficits, infarct size, BBB permeability and expression levels of permeability-related proteins in the brain. It was found that curcumin pretreatment significantly improved neurological scores, decreased infarct size, and protected synaptic remodeling of hippocampal neurons and upregulated the protein expression level of tight junction proteins, ZO-1, occludin and claudin-5 in ischemic rat brains. Furthermore, curcumin pretreatment before stroke was shown to downregulate the phosphorylation of $\mathrm{NF}-\kappa \mathrm{B}$ and MMP-9, which are central mediators of inflammation. The results from the present study indicated that curcumin pretreatment ameliorated ischemic stroke injury by protecting BBB integrity and synaptic remodeling, as well as inhibiting inflammatory responses.
\end{abstract}

Correspondence to: Dr Xuemei Zong or Professor Shuqun Hu, Jiangsu Provincial Institute of Health Emergency, Xuzhou Medical University, 99 Huaihai Road, Xuzhou, Jiangsu 221002, P.R. China E-mail: xuemeizong@gmail.com

E-mail: hushuqun88@126.com

*Contributed equally

Key words: curcumin, ischemic stroke, blood-brain barrier, inflammation, tight junction

\section{Introduction}

Stroke, a common neurological disorder associated with a high risk of disability and mortality (1), disrupts the blood-brain barrier (BBB), where it can induce neuronal injury through brain edema and inflammation (2). There is increasing evidence that inflammation contributed to the progression of ischemia-induced secondary brain damage, leading to aggravated dysregulation of the BBB, as well as cerebral edema $(3,4)$. Thus, identification of novel drugs to protect against ischemic stroke and to understand the underlying mechanisms involved is important to combat this pathology (5).

The BBB consists of a variety of cell types and acts as a border between the brain and the blood circulating through the body (6). It is well-known that BBB impairment is a consequence of ischemic stroke $(7,8)$. Controlling the BBB during stroke has both neuronal and vascular protective effects (9). Previous studies have suggested that NF- $\kappa$ B and MMP-9, which are central mediators of inflammation, play pivotal roles in inflammatory damage following experimental ischemic stroke $(10,11)$.

There are numerous types of MMPs. The MMP that damages the BBB following stroke is gelatinase MMP-9 (12). Under normal, steady state conditions, MMP-9 protein expression levels in endothelial cells are low and are present as inactive zymogens. In animals experiencing cerebral ischemia and reperfusion, inflammatory mediators are upregulated and activate leukocytes, which can secrete MMP-9 (13). When MMP-9 protein levels in the endothelial cells are elevated, extracellular matrix components and substrates in the BBB are damaged, primarily through degradation of type IV collagen. Structural damage to the BBB will increase permeability and result in vascular-derived brain edema, and toxic substances are introduced into the BBB and harm brain tissue (14). Notably, NF- $\kappa$ B directly regulates the transcription of MMP-9, and inhibition of $\mathrm{NF}-\kappa \mathrm{B}$ activity reduces the protein expression level of MMP-9, as well as inflammation $(15,16)$. Reduced MMP-9 protein expression in endothelial cells, as well as 
decreased inflammation, reduces BBB disruption and cerebral injury caused by ischemic stroke (17).

Curcumin, a pleiotropic agent extracted from the rhizome of Curcuma longa (18), exerts pharmacological effects against stroke via its anti-oxidative and anti-inflammatory action (19). Previous research has revealed that curcumin could pass through the BBB and significantly decreased water content, infarct size and BBB leakage in a middle cerebral artery occlusion/reperfusion (MCAO/R) rat model (20-23). The post-ischemic neuroprotective effects of curcumin have been investigated; however, the potential mechanisms behind these effects are not fully known.

The aim of the present study was to assess how curcumin affected brain injury, the BBB status and the protein expression level of phosphorylated (p)NF- $\kappa B p 65$ and MMP-9 in a rat $\mathrm{MCAO} / \mathrm{R}$ stroke model. We hypothesized that pretreatment with curcumin would protect $\mathrm{BBB}$ integrity after $\mathrm{MCAO} / \mathrm{R}$ in the brain via its anti-inflammatory effects, including the attenuation of pNF-кBp65 and MMP-9 in endothelial cells and increase the expression level of tight junction proteins.

\section{Materials and methods}

Animal and establishment of the stroke model. A total of 903 -month-old male Sprague-Dawley (SD) rats, weighing 250-280 g were provided by Xuzhou Medical University (Jiangsu, China). The rats were housed in a temperature-controlled $\left(22 \pm 2^{\circ} \mathrm{C}\right)$ setting, including $55 \pm 10 \%$ humidity and a $12 \mathrm{~h}$ light/dark cycle with free access to food and water. All rats were sufficiently adapted to their environments before subjected to surgery. The MCAO/R stroke model used in the present study was established as previously described (24). Briefly, rats were intraperitoneally anesthetized with ketamine $(60 \mathrm{mg} / \mathrm{kg})$ and xylazine $(5 \mathrm{mg} / \mathrm{kg})$. The ventral midline neck was cut to expose the right common (CCA), right internal (ICA) and right external (ECA) carotid arteries. The ECA branches were then ligated. Next, a 4-0 nylon monofilament containing a round tip (Guangzhou Jialing Biotechnology Co., Ltd.) was inserted along the CCA into the ICA. This was performed until resistance was detected. Approximately 90 min following MCAO surgery, the monofilament was removed, followed by reperfusion for $24 \mathrm{~h}$, and 3 and 7 days post-MCAO. The exact same surgery was performed in the sham group rats; however, filament insertion was not performed.

The SD rats were randomized into three individual groups, including the sham $(n=30)$, the MCAO/R with vehicle $(n=30)$ and the MCAO/R plus curcumin groups $(n=30)$. Curcumin (300 mg/kg; Sigma-Aldrich; Merck KGaA) was dissolved in $2 \%$ dimethyl sulfoxide (vehicle) and intraperitoneally administered $30 \mathrm{~min}$ prior to MCAO/R surgery (25). All the experiments were performed following guidelines written by the Institutional Animal Care and Use Committee of China. The studies were also approved by the Ethics Committee for the Use of Experimental Animals at Xuzhou Medical University (assurance nos. 2015-46 and 2015-47).

Assessing neurological deficits. The modified Neurological Severity Scale (mNSS) has a total score of 18 points and is divided into 4 parts as follows: Motion, sensation, balance, and reflex. The score of normal rats was 0 . The higher the score, the more severe the symptoms of neural power defciency. Neurological functions were evaluated $24 \mathrm{~h}$, and 3 and 7 days following MCAO/R using a modified Neurological Severity Scale (mNSS), as previously described (26). This scale includes measurement of balance, reflex, sensory and motor skills. The mNSS is similar to the contralateral neglect tests performed for humans. Neurological functions were measured on a scale from 0 to 18 , where normal was scored as 0 and maximum deficit was scored as 18. A mNSS score of 0 was indicative of the sham group.

Measuring infarct size. The rats were anesthetized $24 \mathrm{~h}$ post-MCAO/R and $2 \mathrm{~mm}$ coronal slices were prepared. The issues were stained at $37^{\circ} \mathrm{C}$ for $30 \mathrm{~min}$ using $2 \%$ solution 2,3 , 5-triphenyltetrazolium chloride (TTC; Sigma-Aldrich; Merck $\mathrm{KGaA}$ ) diluted in PBS. Following which, the tissues were fixed using $4 \%$ paraformaldehyde in a $4^{\circ} \mathrm{C}$ refrigerator overnight. Red staining was associated with undamaged regions after $\mathrm{MCAO} / \mathrm{R}$ and white stained areas revealed regions experiencing an infarct. The relative infarction volume percentage (RIVP) was calculated using the following formula: $\mathrm{RIVP}=$ total infarct area/total area $\mathrm{x} 100 \%$. Infarct size was quantified using ImageJ software 1.8.0 (National Institutes of Health).

Rapid golgi staining. The brains were removed from the rats and processed using the rapid Golgi staining kit (FD Neurotechnologies, Inc.), based on the instructions provided by the manufacturer. Briefly, serial sections $(100-\mu \mathrm{m})$ from the hippocampus were prepared on a freezing microtome and dehydrated in an ascending absolute ethanol series (50, 70 and 90\%), washed in xylene and mounted in neutral balsam. Next, 5 pyramidal neurons extracted from each rat (3 rats/group; 20 brain sections from each group) were measured from area $\mathrm{CA} 1$ of the hippocampus. A camera lucida drawing tube, attached to an Olympus BX51 microscope (x400; Olympus Corporation) was used to select neurons for analyses. To analyze the neurons, the soma center was considered as the reference dot. Total dendritic length and number were measured every $50 \mu \mathrm{m}$.

Evaluation of BBB permeability. To measure BBB permeability, Evans blue (EB) dye was used as a tracer, as previously described (27). Briefly, the rats were injected with $2 \%$ EB solution diluted in normal saline through the tail vein $(2 \mathrm{ml} / \mathrm{kg}$ of body weight; Sigma-Aldrich; Merck KGaA) 24 h post-surgery. The EB dye was allowed to circulate for $2 \mathrm{~h}$. Next, the rats were anesthetized and transcardially perfused using $0.9 \%$ sodium chloride. The brains were removed, divided into left and right hemispheres, then the right hemisphere was immersed in formamide $\left(10 \mathrm{ml} / \mathrm{kg}\right.$; Sigma-Aldrich; Merck KGaA) at $60^{\circ} \mathrm{C}$ for $24 \mathrm{~h}$. Cortical proteins were formamide extracted and centrifuged $(2,500 \mathrm{x} \mathrm{g})$ for $10 \mathrm{~min}$ at $4^{\circ} \mathrm{C}$. A total of $1 \mathrm{ml}$ supernatant was measured in a spectrophotometer at $620 \mathrm{~nm}$ to compare EB content in the brain tissue with standard EB solution.

Immunofluorescence. Following brain perfusion, the tissues were further post-fixed overnight in $4 \%$ paraformaldehyde and $4^{\circ} \mathrm{C}$ refrigerator, then dehydrated in $30 \%$ sucrose. Cerebral 
A
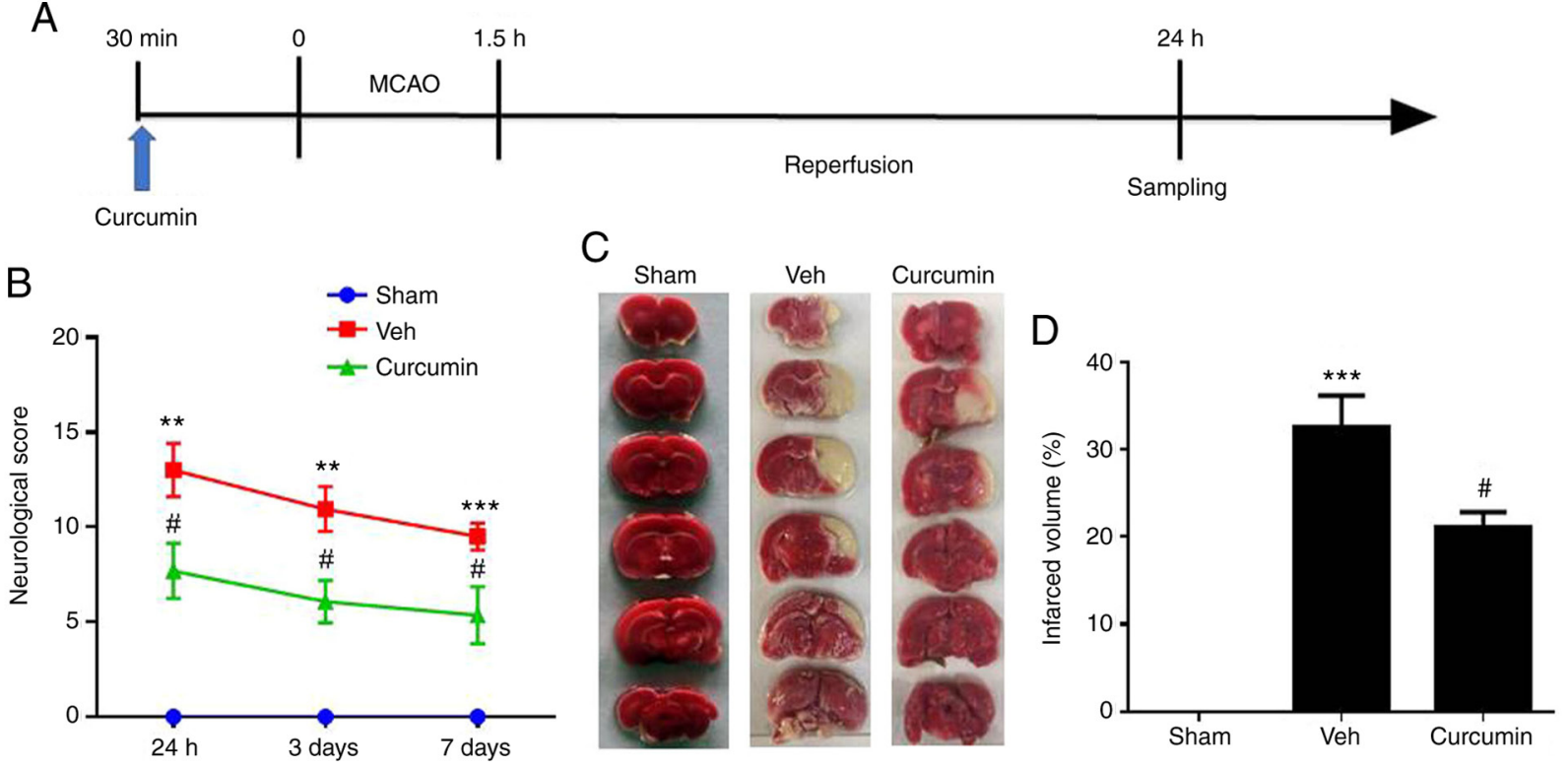

Figure 1. Curcumin pretreatment ameliorates $\mathrm{MCAO} / \mathrm{R}$-induced neurological impairment and early brain injury in rats. (A) Schematic representing the experimental protocol used for measuring the effects of curcumin pretreatment on brain injury. (B) Neurological Severity Scale scores in the different rat groups were evaluated at $24 \mathrm{~h}$, and 3 and 7 days following reperfusion. (C) Rat brains were sliced and stained using 2,3,5-triphenyltetrazolium chloride $24 \mathrm{~h}$ following MCAO/R and (D) the percentage of the relative infarct size was calculated. The data are presented as the mean \pm SEM. $n=6 .{ }^{* * *} \mathrm{P}<0.01,{ }^{* * * *} \mathrm{P}<0.001 \mathrm{vs}$. sham group, ${ }^{,} \mathrm{P}<0.05$ vs. $\mathrm{MCAO} / \mathrm{R}$ vehicle group. Veh, $\mathrm{MCAO} / \mathrm{R}$ vehicle group; $\mathrm{MCAO} / \mathrm{R}$, middle cerebral artery occlusion/reperfusion.

peri-ischemic cortices were sliced into $20-\mu \mathrm{m}$ slices and washed in PBS. The slices were then incubated in blocking solution $0.1 \%$ Triton X-100 in $0.1 \mathrm{M}$ PBS, $10 \%$ bovine serum albumin (cat. no. Pro-422, pH 7.6; Prospec-Tany Technogene Ltd.) in a $4^{\circ} \mathrm{C}$ refrigerator for $2 \mathrm{~h}$, followed by overnight incubation at $4^{\circ} \mathrm{C}$ with anti-ZO-1 (1:100; cat. no. ab96587; Abcam) and anti-claudin-5 (1:100; cat. no. 352588; Thermo Fisher Scientific, Inc.) antibodies. After washing in PBS, the tissues were incubated with goat biotin-conjugated anti-cat $\operatorname{IgG}$ antibody (1:10,000, cat. no. LS-C68537; LifeSpan BioSciences, Inc.) and counterstained with 4',6-diamidino-2-phenylindole (Beyotime Institute of Biotechnology). Lastly, the sections were analyzed using a spectral confocal microscope DMI6000 (Leica Microsystems GmbH).

Western blot analysis. Peri-ischemic cortical proteins were extracted from the rat brains by homogenizing the tissues in RIPA lysis buffer (cat. no. P0013B; Beyotime Institute of Biotechnology), supplemented with protease and phosphatase inhibitors. The samples were centrifuged at 7,000 x g (EP5417R; Eppendorf) for $20 \mathrm{~min}$ at $4^{\circ} \mathrm{C}$. A BCA assay kit (Thermo Fisher Scientific, Inc.) was used to measure the concentration of each sample. Nuclear and cytoplasmic extraction kits (cat. no. 78833; Thermo Fisher Scientific, Inc.) for nuclear protein extraction. An equal amount $(0.06 \mathrm{mg})$ of protein was separated using 6 and 10\% SDS-PAGE before being transferred to a PVDF membrane (EMD Millipore). Membranes were blocked with 5\% skimmed milk for $90 \mathrm{~min}$. Following blocking, the membranes were incubated overnight at $4^{\circ} \mathrm{C}$ with anti-pNF- $\mathrm{Bp} 65$ (1:1,000; cat. no ab222494; Abcam), anti-NF-кBp65 (1:1,000; cat. no. ab228497; Abcam), anti-MMP-9 (1:1,000; cat. no. ab38898; Abcam), anti- $\beta$-actin (1:5,000; cat. no. ab11003; ABclonal Biotech Co., Ltd.), anti-occludin (1:1,000; cat. no. ab216327; Abcam), anti-claudin-5 (1:500; cat. no. 35288; Thermo Fisher Scientific, Inc.) and anti-ZO-1 (1:1,000; cat. no. ab96587; Abcam) antibodies. After several washes with TBS/0.1\% Tween-20 and incubation with the goat anti-cat IgG antibody (1:10,000, LS-C68537, LSBio) for $2 \mathrm{~h}$ at room temperature, the immuno-reactive bands were visualized using an ECL kit (Beyotime Institute of Biotechnology). The protein bands were then analyzed using ImageJ software version 1.8.0 (National Institutes of Health).

Statistical analysis. All the data are presented as the mean \pm SEM. Shapiro-Wilk normality test was used to determine if the data was normally distributed. Statistically significant differences were analyzed using one-way ANOVA for multiple groups or an unpaired Student's t-test for comparisons of 2 groups were applied. All statistical analyses were performed using GraphPad prism v5 (GraphPad Software, Inc.). $\mathrm{P}<0.05$ was considered to indicate a statistically significant difference.

\section{Results}

Curcumin ameliorates early brain injury and improves neurological performance in MCAO/R rats. A schematic diagram representing experimental procedures is shown in Fig. 1A (reperfusion was performed $24 \mathrm{~h}$, and 3 and 7 days following $\mathrm{MCAO} / \mathrm{R}$ ). As depicted in Fig. 1B, vehicle-treated $\mathrm{MCAO} / \mathrm{R}$ rats exhibited poor and reduced neurological function as compared with that in the sham group. Based on the $\mathrm{mNSS}$ scale, the $\mathrm{MCAO} / \mathrm{R}$ rats treated with curcumin showed a significant improvement in neurological performance at all the measured time points ( $24 \mathrm{~h}$, and 3 and 7 days following reperfusion), where the most optimal effect was noted $24 \mathrm{~h}$ post-surgery $(\mathrm{P}<0.05$; Fig. 1B). As the lowest mNSS score 

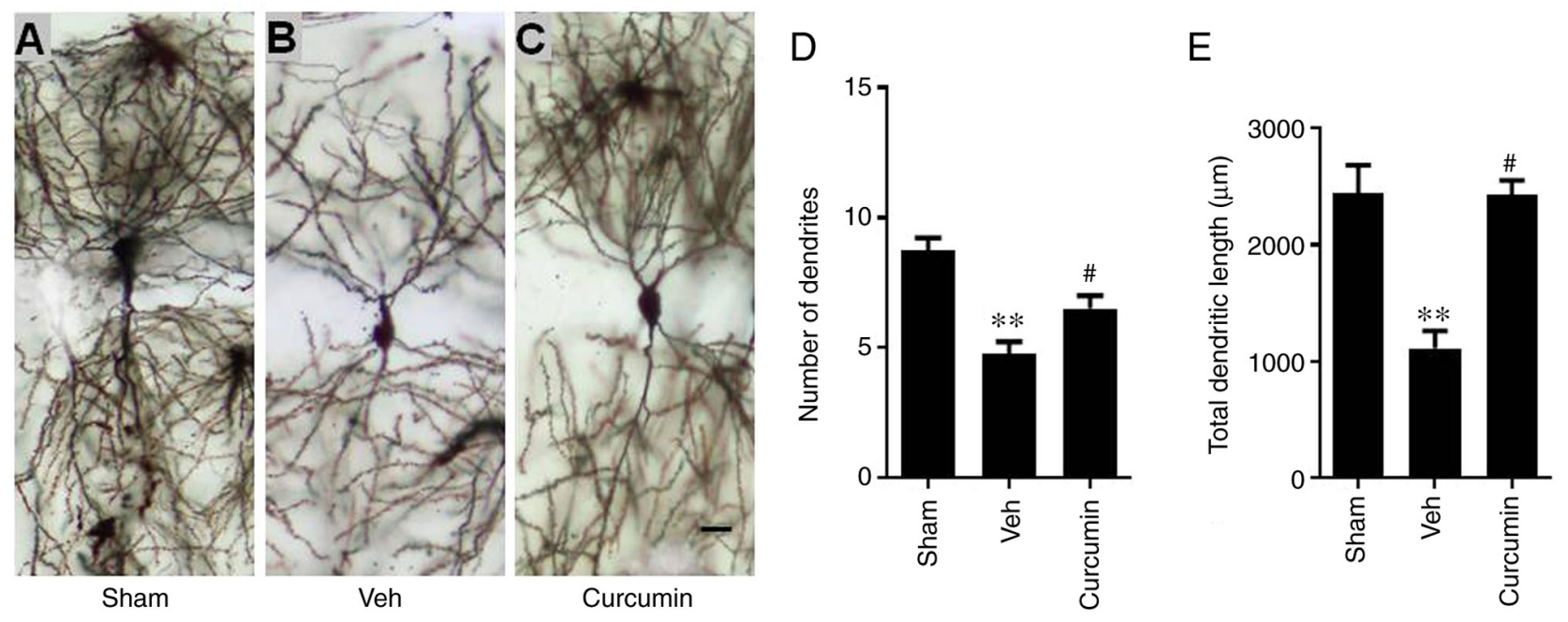

Figure 2. Protective effects of curcumin on hippocampus neuronal remodeling against MCAO/R. Representative images of golgi stained hippocampus pyramidal neurons in the (A) sham, (B) vehicle-treated MCAO/R and (C) curcumin-treated MCAO/R groups. Scale bar, $10 \mu \mathrm{m}$. Quantitative analyses of the (D) numbers of dendrites and (E) total dendritic length. The data are presented as the mean $\pm \mathrm{SEM}$. $\mathrm{n}=6$. ${ }^{* *} \mathrm{P}<0.01 \mathrm{vs}$. sham group, ${ }^{\#} \mathrm{P}<0.05 \mathrm{vs}$. MCAO/R vehicle group. Veh, MCAO/R vehicle group; $\mathrm{MCAO} / \mathrm{R}$, middle cerebral artery occlusion/reperfusion.

was observed $24 \mathrm{~h}$ following reperfusion, this time point was selected for subsequent experiments.

TTC staining was used to measure infarct volume $24 \mathrm{~h}$ post-reperfusion. The results showed an absence of infarct in the sham group, but significant ischemic injury in the $\mathrm{MCAO} / \mathrm{R}$ group compared with that in the sham group $(\mathrm{P}<0.001$; Fig. 1C). However, curcumin pre-treatment significantly reduced infarct size compared with that in the vehicle treated $\mathrm{MCAO} / \mathrm{R}$ group $(\mathrm{P}<0.05$; Fig. 1D). Therefore, it was concluded that pretreatment with curcumin protected rats against cerebral injury and improved neurological deficits in $\mathrm{MCAO} / \mathrm{R}$ rats.

Curcumin protects synaptic remodeling of hippocampal neurons against MCAO/R. As shown in Fig. 2A-E, neurons were selected according to the following criteria: The body and dendrites of the neuron were completely impregnated, the neuron was relatively separated from the surrounding neurons and the neuron was located in the hippocampal CA1 area. Golgi staining and quantitative analysis indicated that rats in the vehicle-treated $\mathrm{MCAO} / \mathrm{R}$ group showed a significant decrease in the number of hippocampal dendrites, as well as total dendritic length as compared with that in the sham group $(\mathrm{P}<0.01)$. The rats that were treated with curcumin before $\mathrm{MCAO} / \mathrm{R}$ had significantly reduced $\mathrm{MCAO} / \mathrm{R}$-induced atrophy in the hippocampal neurons, as there was an increase in both dendrite number and length $(\mathrm{P}<0.05)$. These data revealed that curcumin could protect hippocampal neuron remodeling when subjected to $\mathrm{MCAO} / \mathrm{R}$.

Curcumin pretreatment protects against $B B B$ permeability induced by $M C A O / R$. The brain sections were viewed using a EB tracer, as shown in Fig. 3A. Leakage of EB dye was significantly attenuated in the curcumin group compared with that in the $\mathrm{MCAO} / \mathrm{R}$ vehicle group. Levels of formamide extracted from EB dye were further analyzed. Fig. 3B demonstrated that $\mathrm{MCAO} / \mathrm{R}$ induced significant $\mathrm{EB}$ extravasation as compared with that in the sham group at $24 \mathrm{~h}(\mathrm{P}<0.001)$.
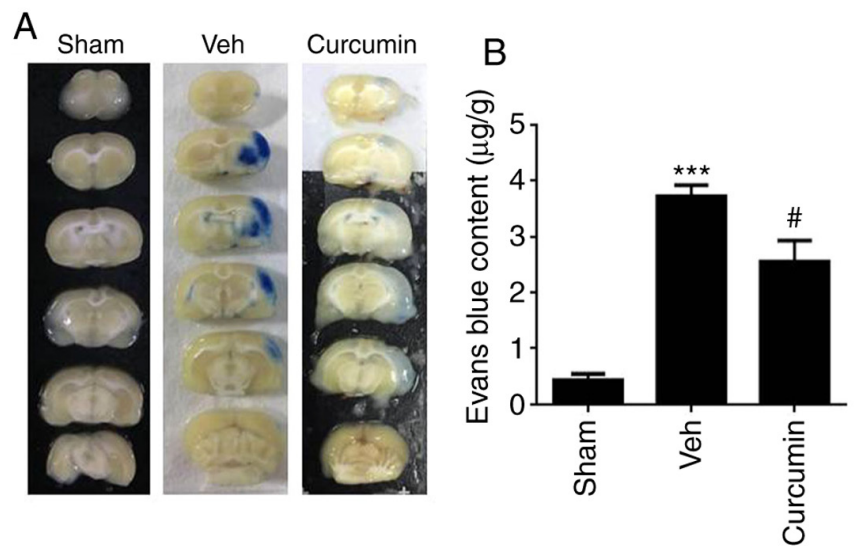

Figure 3. Curcumin protects BBB integrity impaired by ischemic reperfusion. (A) Representative images showing leakage of Evans blue dye $24 \mathrm{~h}$ following $\mathrm{MCAO} / \mathrm{R}$. (B) Quantified levels of formamide extracted Evans blue from brain tissue. The data are presented as the mean \pm SEM. $n=6{ }^{*}{ }^{* * *} \mathrm{P}<0.001$ vs. sham group, ${ }^{\#} \mathrm{P}<0.05$ vs. $\mathrm{MCAO} / \mathrm{R}$ vehicle group. Veh, $\mathrm{MCAO} / \mathrm{R}$ vehicle group; $\mathrm{MCAO} / \mathrm{R}$, middle cerebral artery occlusion/reperfusion.

Curcumin pretreatment significantly reduced EB extravasation levels $(\mathrm{P}<0.05)$ compared with that in the vehicle-treated $\mathrm{MCAO} / \mathrm{R}$ group. This indicated that curcumin could protect $\mathrm{MCAO} / \mathrm{R}$ rats from disrupted $\mathrm{BBB}$ permeability resulting from ischemic damage.

Curcumin suppresses the MCAO/R-induced inflammatory response. The inflammatory response is activated as a result of BBB injury (28); therefore, it was determined whether curcumin pretreatment could affect the protein expression levels of known inflammatory mediators. Following reperfusion for $24 \mathrm{~h}$, the protein expression levels of both pNF-кBp65 and MMP-9 were increased. However, when exposed to curcumin pretreatment, there was a significant reduction in the expression levels of both pNF- $\kappa \mathrm{Bp} 65(\mathrm{P}<0.01$; Fig. 4A) and MMP-9 $(\mathrm{P}<0.05$; Fig. 4B) compared with the vehicle groups. Taken together, pretreatment with curcumin suppressed the 
A
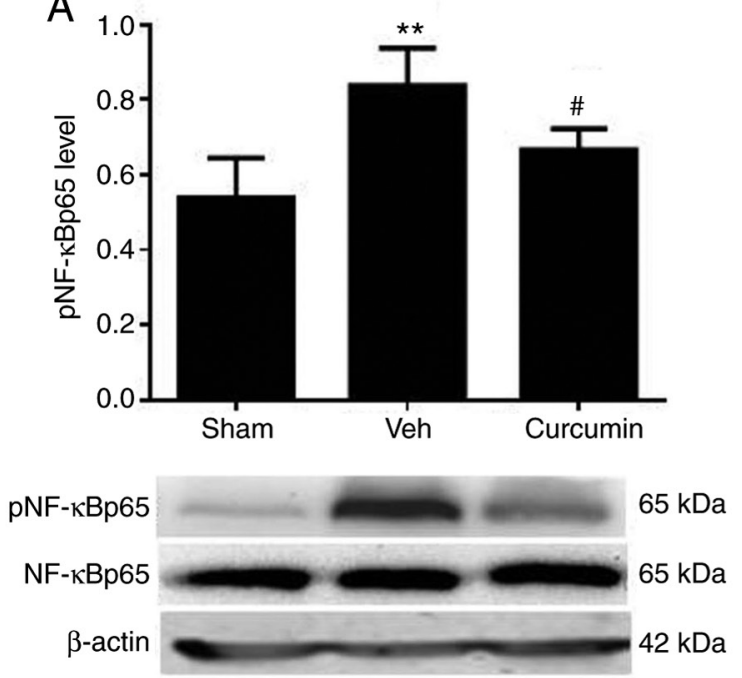

$\mathrm{B}$
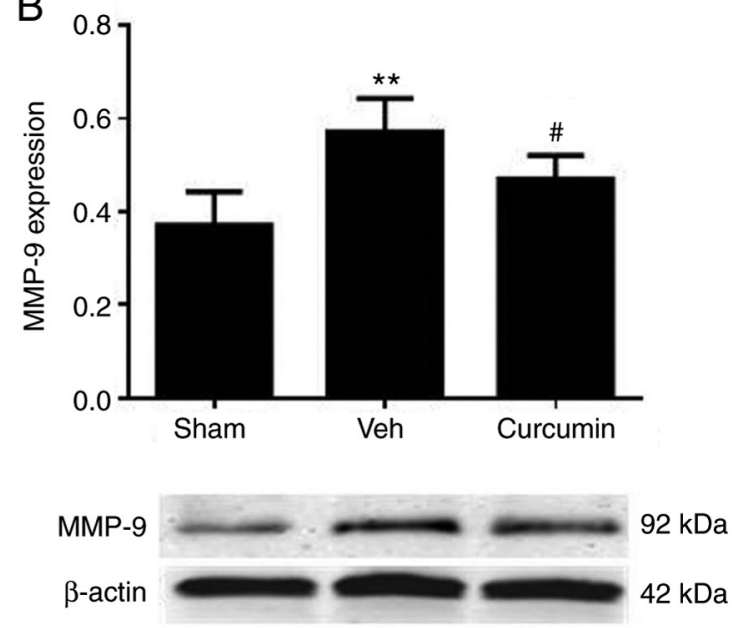

Figure 4. Curcumin reduces MCAO/R-induced protein expression levels of pNF- $\kappa$ Bp65, NF- $\kappa$ Bp65 and MMP-9. Representative western blots and densito-

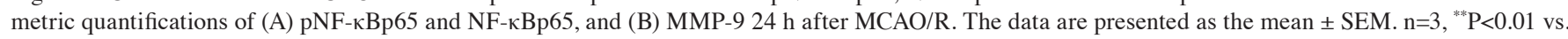
sham group, ${ }^{\#} \mathrm{P}<0.05$ vs. $\mathrm{MCAO} / \mathrm{R}$ vehicle group. Veh, $\mathrm{MCAO} / \mathrm{R}$ vehicle group; $\mathrm{MCAO} / \mathrm{R}$, middle cerebral artery occlusion/reperfusion; $\mathrm{p}$, phosphorylated.

inflammatory response, which was revealed by the reduction of key inflammatory mediators.

Curcumin preserves in-situ protein expression level of claudin-5 and ZO-1 in MCAO/R rats. Subsequently, the effects of curcumin pretreatment on BBB permeability were further investigated to confirm the earlier result. Immunohistochemistry, using claudin-5 and ZO-1 antibodies, was performed on the tissues extracted from the rats in the sham and $\mathrm{MCAO} / \mathrm{R}$ groups, $24 \mathrm{~h}$ following reperfusion. As seen in Fig. 5A and B, claudin-5 and ZO-1 protein expression levels were markedly decreased in the cortex of the rats, $24 \mathrm{~h}$ following $\mathrm{MCAO} / \mathrm{R}$ injury as compared with that in the sham group. However, in the group of curcumin pretreated rats, both claudin-5 and ZO-1 expression levels were increased as compared with that in the MCAO/R rats treated with vehicle. These data suggested that curcumin could reverse the attenuation of claudin-5 and ZO-1, which typically occurs when the BBB is disrupted following ischemic brain injury.

Curcumin treatment prevents the degradation of $B B B$ tight junction proteins. Lastly it was investigated how curcumin pretreatment affected BBB integrity and permeability in rats, which had experienced a stroke. The protein expression levels of tight junction proteins, ZO-1, occludin and claudin-5 were analyzed, which play an important role in BBB integrity (29). Western blot analysis followed by densitometry analysis revealed that $\mathrm{MCAO} / \mathrm{R}$ induced significant reduction of ZO-1, occludin and claudin-5 protein expression levels, $24 \mathrm{~h}$ following reperfusion as compared with that in the sham rats. Notably, the protein expression levels of these tight junction proteins were significantly increased following curcumin pretreatment $(\mathrm{P}<0.05$; Fig. 6A-C). Therefore, curcumin may protect the integrity of the BBB by inhibiting the degradation of tight junction proteins induced by $\mathrm{MCAO} / \mathrm{R}$.

\section{Discussion}

In the present study, the effects of curcumin on reperfusion-induced $\mathrm{BBB}$ disruption in a rat model of $\mathrm{MCAO} / \mathrm{R}$ was investigated. The results revealed that pretreatment with curcumin protected rats from $\mathrm{MCAO} / \mathrm{R}$ and the resulting neurological deficits, reduced infarct size, as well as nerve injury. To obtain a mechanistic insight behind these effects, it was identified that curcumin repressed the inflammatory response and restored levels of tight junction proteins early enough to protect against BBB deterioration.

Curcumin belongs to a class of yellow polyphenols derived from turmeric medicinal plants (25). A previous study has shown that curcumin exerted an array of biological functions, including anti-tumor, anti-immunity, anti-oxidation, anti-fibrosis and anti-inflammatory effects (30). Recently, a study has indicated that curcumin harbored protective effects against neuronal damage following cerebral ischemia (31). In addition, previous studies have shown that following curcumin treatment, neurological scores were improved, early brain injury was ameliorated, and $\mathrm{NF}-\kappa \mathrm{B}$ and MMP-9 protein expression levels in brain tissue of injured rats were decreased $(25,32,33)$. Furthermore, both the number of hippocampal neuronal dendrites and total dendritic length were increased and EB content in the brain tissue was significantly decreased (34-36). The results from the present study provides additional results by revealing the beneficial effects of curcumin pretreatment against $\mathrm{MCAO} / \mathrm{R}$ injury. Abnormal hippocampal neurotransmitters may affect neural plasticity, which may be associated with the pathophysiology of ischemic cerebral infarction (37). In the present study, it was found that in the MCAO/R rat model, hippocampal neuronal remodeling could be significantly disrupted following ischemia and reperfusion. It was also confirmed that the administration of curcumin protected the BBB structure, inhibited the inflammatory response of the hippocampus, and remodeled hippocampal neurons against 
A

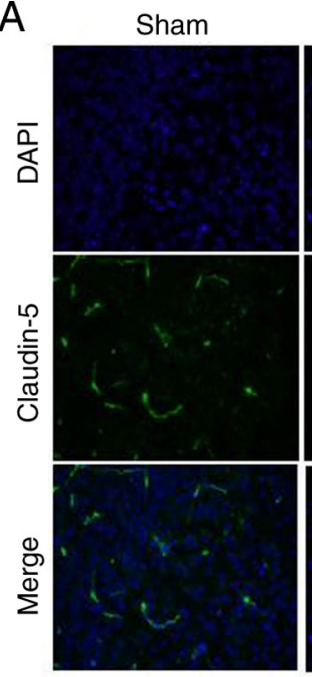

Veh

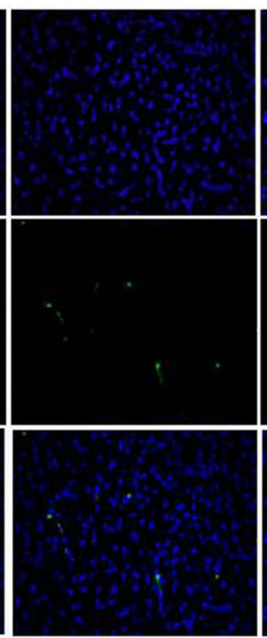

Curcumin

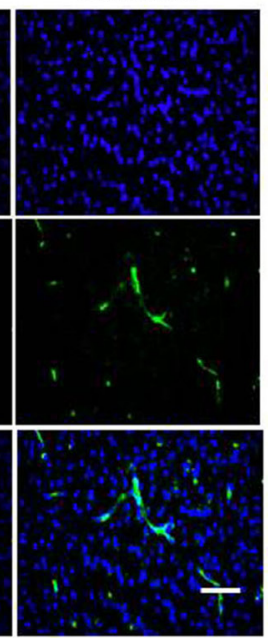

B

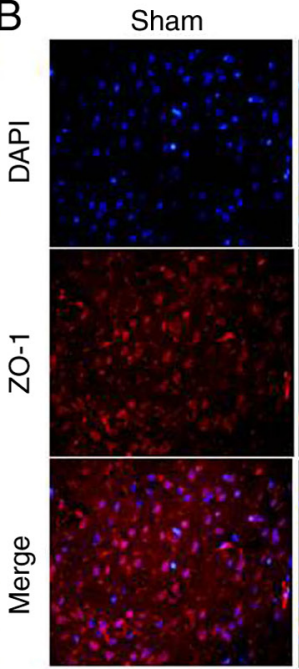

Veh

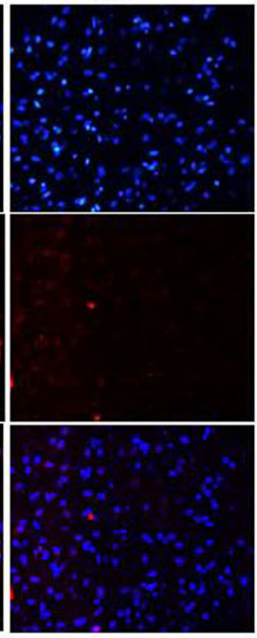

Curcumin

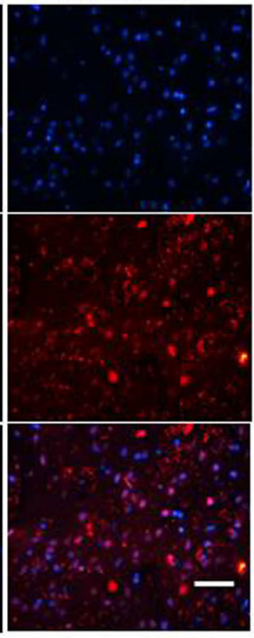

Figure 5. Curcumin reverses the attenuation of tight junction proteins claudin-5 and ZO-1 induced by MCAO/R. Representative in situ expression of (A) claudin-5 (green) and (B) ZO-1 (red), and merged with DAPI counter staining (blue) in the peri-infarct region from sham-operated, MCAO/R vehicle and curcumin treated animals. Scale bar, $50 \mu \mathrm{m}$. Veh, MCAO/R vehicle group; MCAO/R, middle cerebral artery occlusion/reperfusion; DAPI, 4',6-diamidino-2-phenylindole.
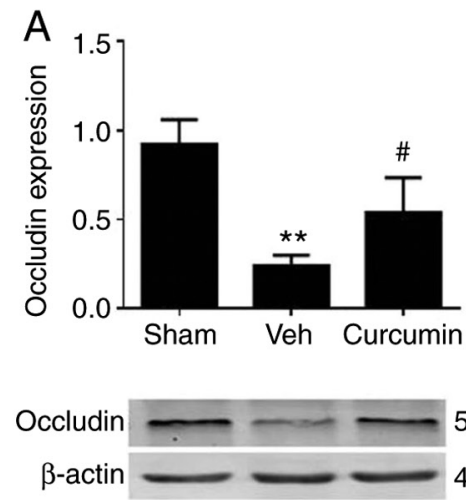

B

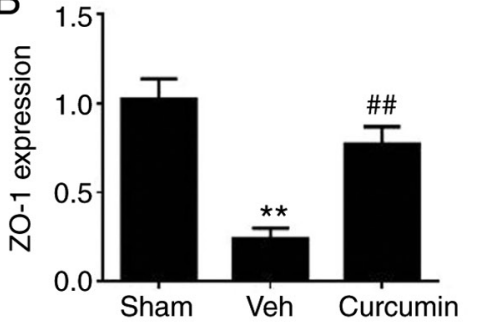

$56 \mathrm{kDa}$
$42 \mathrm{kDa}$

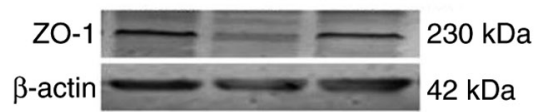

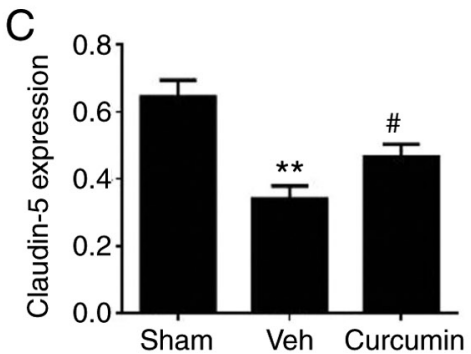

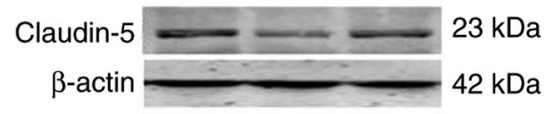

Figure 6. Curcumin inhibits the degradation of tight junction proteins. Representative western blots and quantitative analyses showing the protein expression levels of (A) occludin, (B) ZO-1 and (C) claudin-5 in the rat brain tissues from the 3 experimental groups. The data are presented as the mean \pm SEM. $\mathrm{n}=3$. ${ }^{* *} \mathrm{P}<0.01$ vs. sham group, ${ }^{\#} \mathrm{P}<0.05$ vs.MCAO/R vehicle group. ${ }^{\# \#} \mathrm{P}<0.01$ vs. MCAO/R vehicle group. Veh, MCAO/R vehicle group; MCAO/R, middle cerebral artery occlusion/reperfusion.

MCAO/R (37-39). The protective effect of curcumin on hippocampal neurons provides evidence of its advantage in the treatment of ischemic stroke.

In most unstimulated cells, the inactive p65 component of NF- $\kappa \mathrm{B}$ remains in the cytoplasm as a dimer, which can bind to IkB. The protein expression levels of tumor necrosis factor (TNF)- $\alpha$ and IL-1 $\beta$ were increased at the early stages of the inflammatory response following cerebral ischemia and reperfusion $(30,40)$. As the initiating factors of the inflammatory response, both TNF- $\alpha$ and IL- $1 \beta$ can stimulate leukocytes and activate the c signaling pathway $(33,41)$. The IkB protein is phosphorylated and targeted for degradation via the proteasome pathway, where the p65 component of $\mathrm{NF}-\kappa \mathrm{B}$ is translocated to the nucleus to form $\mathrm{pNF}-\kappa \mathrm{Bp} 65$ and increases the secretion of adhesion molecules via microvascular endothelial cells and leukocytes (42). Leukocytes can adhere to vascular endothelial cells and produce neutrophil-derived oxidant and MMP-9 (13), resulting in
BBB damage from MMP-9 $(25,32)$. Previous studies have also showed that MMP-9 protein was expressed in endothelial cells and the main cellular source of brain MMP-9 are brain microvascular endothelial cells in the initial phase following focal cerebral ischemia $(43,44)$. Based on this, it could be concluded that inhibition of $N F-\kappa B$ activation could reduce ischemia-reperfusion injury in rats (45). The protein expression levels of $\mathrm{pNF}-\kappa \mathrm{Bp} 65$ were analyzed in the nucleus. The results showed that curcumin pretreatment effectively reduced the protein expression levels of pNF- $\kappa$ Bp 65 , inhibited the transcriptional activity of NF- $\kappa \mathrm{B}$ in cerebral ischemia/reperfusion ( $/ / R)$ and may have a strong anti-inflammatory effect.

Activation of MMP-9 leads to the degradation of key proteins located in the cerebral blood vessels, mainly through the degradation of type IV collagen. The extracellular matrix components and substrates in the BBB are destroyed, resulting in the extravasation of water in the capillaries and plasma 
proteins in the peripheral blood, resulting in an increase of water in the cell space and formation of vascular-derived brain edema. There is an introduction of toxic substances into the $\mathrm{BBB}$ and damage to brain tissue, which destroys the integrity of the vascular structure $(43,46,47)$. Previously, the association between MMP-9 and ischemic brain injury has gained interest $(46,47)$. When an inflammatory reaction occurs in the body, activated white blood cells secrete substances that can be toxic, including IL-8, TNF, MMPs, nitric oxide and reactive oxygen species. Among these, MMP-9 is a key protein, which causes damage to the BBB. Inhibition of MMP-9 has been reported to prevent damage to the brain by maintaining BBB integrity in elderly humans (48). In the present study, curcumin pretreatment was found to significantly reduce MMP-9 protein expression levels induced following MCAO/R. This is consistent with previous reports showing that MMP-9 inhibition mediated $\mathrm{BBB}$ protection in a mouse model of ischemic stroke (16). Therefore, it is reasonable to conclude that curcumin pretreatment could reduce $\mathrm{BBB}$ damage at least via the reduction of MMP-9 protein expression level to protect against damage from cerebral ischemia.

The BBB is composed of highly selective tight junctions between endothelial cells, made primarily of the membrane-associated accessory proteins, including occludin, ZO-1 and claudin-5 $(4,49,50)$. The results from the present study revealed that pretreatment with curcumin blocked the decrease of tight junction protein expression, which is instrumental in maintaining BBB integrity and function.

In summary, the present study revealed that pretreatment with curcumin prior to stroke could inhibit the central pro-inflammatory mediator $\mathrm{NF}-\kappa \mathrm{B}$, reduce the protein expression level of MMP-9 and attenuate BBB damage, indicating its neuroprotective effects. These data provide novel targets to investigate its protective effects underlying cerebral ischemic injury and provide a new direction to determine therapeutics for brain insults by restoring the BBB.

\section{Acknowledgements}

Not applicable.

\section{Funding}

This research was supported by the Jiangsu Provincial Commission of Health and Family Planning, Genera Programs (grant. no. H201527), Open Project Program of Jiangsu Key Laboratory of Anesthesiology (grant no. KJS1704) and Jiangsu Social Development Foundation (grant no.BE2017641).

\section{Availability of data and materials}

The datasets used and/or analyzed during the current study are available from the corresponding author on reasonable request.

\section{Author's contributions}

$\mathrm{XZ}$ and SH conceived the design of the study. SW and TG performed the experiments and analyzed the data. TG, WQ, YL, JG and CL performed the experiments and analyzed the data. YS and BY collected the data. YS, BY, SH and XZ performed data analysis and/or wrote part of the paper. SW, $\mathrm{XZ}$ and SH confirm the authenticity of all the raw data. All authors read and approved the final manuscript.

\section{Ethics approval and consent to participate}

All the experiments were performed following guidelines written by the Institutional Animal Care and Use Committee of China. The studies were also approved by the Ethics Committee for the Use of Experimental Animals at Xuzhou Medical University (assurance nos. 2015-46 and 2015-47).

\section{Patient consent for publication}

Not applicable.

\section{Competing interests}

The authors declare that they have no competing interests.

\section{References}

1. Bai X, Zhang YL and Liu LN: Inhibition of TRIM8 restrains ischaemia-reperfusion-mediated cerebral injury by regulation of $\mathrm{NF}-\kappa \mathrm{B}$ activation associated inflammation and apoptosis. Exp Cell Res 388: 111818, 2020.

2. Li W, Suwanwela NC and Patumraj S: Curcumin prevents reperfusion injury following ischemic stroke in rats via inhibition of NF- $\kappa$ B, ICAM-1, MMP-9 and caspase-3 expression. Mol Med Rep 47: 4710-4720, 2017.

3. Wang L, Geng J, Qu M, Yuan F, Wang Y, Pan J, Li Y, Ma Y, Zhou P, Zhang Z and Yang GY: Oligodendrocyte precursor cells transplantation protects blood-brain barrier in a mouse model of brain ischemia via Wnt/ $\beta$-catenin signaling. Cell Death Dis 11: 9, 2020

4. Beard RS Jr, Reynolds JJ and Bearden SE: Hyperhomocysteinemia increases permeability of the blood-brain barrier by NMDA receptor-dependent regulation of adherens and tight junctions. Blood 118: 2007-2014, 2011.

5. Yang E, Cai Y, Yao X, Liu J, Wang Q, Jin W, Wu Q, Fan W, Qiu L, Kang C and Wu J: Tissue plasminogen activator disrupts the blood-brain barrier through increasing the inflammatory response mediated by pericytes after cerebral ischemia. Aging (Albany NY) 11: 10167-10182, 2019.

6. Cao C, Zhou J, Wu X, Qian Y, Hong Y, Mu J, Jin L, Zhu C and Li S: Activation of CRHR1 contributes to cerebral endothelial barrier impairment via cPLA2 phosphorylation in experimental ischemic stroke. Cell Signal 66: 109467, 2020.

7. Liu ZJ, Liu W, Liu L, Xiao C, Wang Y and Jiao JS: Curcumin protects neuron against cerebral ischemia-induced inflammation through improving PPAR-Gamma function. Evid Based Complement Alternat Med 2013: 470975, 2013.

8. Zhang DD, Jin C, Zhang YT, Gan XD, Zou MJ, Wang YY, Fu WL, Xu T, Xing WW, Xia WR and Xu DG: A novel IL-1RA-PEP fusion protein alleviates blood-brain barrier disruption after ischemia-reperfusion in male rats. J Neuroinflammation 15: 16, 2018.

9. Guo P, Jin Z, Wu H, Li X, Ke J, Zhang Z and Zhao Q: Effects of irisin on the dysfunction of blood-brain barrier in rats after focal cerebral ischemia/reperfusion. Brain Behav 9: e01425, 2019.

10. Bai X, Zhang X, Chen L, Zhang J, Zhang L, Zhao X, Zhao T and Zhao Y: Protective effect of naringenin in experimental ischemic stroke: Down-regulated NOD2, RIP2, NF-кB, MMP-9 and up-regulated claudin-5 expression. Neurochem Res 39: 1405-1415, 2014.

11. Zhang J, Fu B, Zhang X, Chen L, Zhang L, Zhao X, Bai X, Zhu C, Cui L and Wang L: Neuroprotective effect of bicyclol in rat ischemic stroke: Down-regulates TLR4, TLR9, TRAF6, NF- $\mathrm{B}, \mathrm{MMP}-9$ and up-regulates claudin-5 expression. Brain Res 1528: 80-88, 2013. 
12. Vandooren J, Van Damme J and Opdenakker G: On the structure and functions of gelatinase B/matrix metalloproteinase-9 in neuroinflammation. Prog Brain Res 214: 193-206, 2014.

13. Turner RJ and Sharp FR: Implications of MMP9 for blood brain barrier disruption and hemorrhagic transformation following ischemic stroke. Front Cell Neurosci 10: 56, 2016.

14. Riabinska A, Zille M, Terzi MY, Cordell R, Nieminen-Kelhä M, Klohs J and Piña AL: Pigment epithelium-derived factor improves paracellular blood-brain barrier integrity in the normal and ischemic mouse brain. Cell Mol Neurobiol 40: 751-764, 2020

15. Song Y, Yang Y, Cui Y, Gao J, Wang K and Cui J: Lipoxin A4 methyl ester reduces early brain injury by inhibition of the nuclear factor Kappa B (NF- $\mathrm{B})$-dependent matrix metallopeptidase 9 (MMP-9) pathway in a rat model of intracerebral hemorrhage. Med Sci Monit 25: 1838-1847, 2019.

16. Ludewig P, Sedlacik J, Gelderblom M, Bernreuther C, Korkusuz Y, Wagener C, Gerloff C, Fiehler J, Magnus T and Horst AK: Carcinoembryonic antigen-related cell adhesion molecule 1 inhibits MMP-9-mediated blood-brain-barrier breakdown in a mouse model for ischemic stroke. Circ Res 113: 1013-1022, 2013

17. Li XF, Zhang XJ, Zhang C, Wang LN, Li YR, Zhang Y, He TT, Zhu XY, Cui LL and Gao BL: Ulinastatin protects brain against cerebral ischemia/reperfusion injury through inhibiting MMP-9 and alleviating loss of ZO-1 and occludin proteins in mice. Exp Neurol 302: 68-74, 2018.

18. Ding R, Feng L, He L, Chen Y, Wen P, Fu Z, Lin C, Yang S, Deng X, Zeng J and Sun G: Peroxynitrite decomposition catalyst prevents matrix metalloproteinase- 9 activation and neurovascular injury after hemoglobin injection into the caudate nucleus of rats. Neuroscience 297: 182-193, 2015.

19. Han L, Liu DL, Zeng QK, Shi MQ, Zhao LX, He Q, Kuang X and Du JR: The neuroprotective effects and probable mechanisms of Ligustilide and its degradative products on intracerebral hemorrhage in mice. Int Immunopharmacol 63: 43-57, 2018.

20. Kumari A, Singh DK, Dash D and Singh R: Intranasal curcumin protects against LPS-induced airway remodeling by modulating toll-like receptor-4 (TLR-4) and matrixmetalloproteinase-9 (MMP-9) expression via affecting MAP kinases in mouse model. Inflammopharmacology 27: 731-748, 2019.

21. Wang YF, Gu YT, Qin GH, Zhong L and Meng YN: Curcumin ameliorates the permeability of the blood-brain barrier during hypoxia by upregulating heme oxygenase- 1 expression in brain microvascular endothelial cells. J Mol Neurosci 51: 344-351, 2013.

22. Yavarpour-Bali $\mathrm{H}$, Ghasemi-Kasman $\mathbf{M}$ and irzadeh $\mathbf{M}$ : Curcumin-loaded nanoparticles: A novel therapeutic strategy in treatment of central nervous system disorders. Int J Nanomedicine 14: 4449-4460, 2019.

23. Tsai YM, Chien CF, Lin LC and Tsai TH: Curcumin and its nano-formulation: The kinetics of tissue distribution and blood-brain barrier penetration. Int J Pharm 416: 331-338, 2011

24. Wang Y, Luo J and Li SY: Nano-curcumin simultaneously protects the blood-brain barrier and reduces M1 microglial activation during cerebral ischemia-reperfusion injury. ACS Appl Mater Interfaces 11: 3763-3770, 2019.

25. Bavarsad K, Barreto GE, Hadjzadeh MA and Sahebkar A Protective effects of curcumin against ischemia-reperfusion injury in the nervous system. Mol Neurobiol 56: 1391-1404, 2018

26. Xue X, Wang H and Su J: Inhibition of MiR-122 decreases cerebral ischemia-reperfusion injury by upregulating DJ-1-phosphatase and tensin homologue deleted on chromosome 10 (PTEN)/Phosphonosinol-3 kinase (PI3K)/AKT. Med Sci Monit 26: e915825, 2020.

27. Guo T, Wang Y, Guo Y, Wu S, Chen W, Liu N and Geng D: 1,25-D protects from cerebral ischemia by maintaining BBB permeability via PPAR- $\gamma$ activation. Front Cell Neurosci 12: 480, 2018.

28. Danielson M, Reinsfelt B, Westerlind A, Zetterberg H, Blennow K and Ricksten SE: Effects of methylprednisolone on blood-brain barrier and cerebral inflammation in cardiac surgery-a randomized trial. J Neuroinflammation 15: 283, 2018.

29. Jiao H, Wang Z, Liu Y, Wang P and Xue Y: Specific role of tight junction proteins claudin-5, occludin, and $\mathrm{ZO}-1$ of the blood-brain barrier in a focal cerebral ischemic insult. J Mol Neurosci 44: 130-139, 2011

30. Sikora E, Scapagnini G and Barbagallo M: Curcumin, inflammation, ageing and age-related diseases. Immun Ageing 7: 1, 2010.

31. Huang L, Chen C, Zhang X, Li X, Chen Z, Yang C, Liang X, Zhu G and Xu Z: Neuroprotective effect of curcumin against cerebral ischemia-reperfusion via mediating autophagy and inflammation. J Mol Neurosci 64: 129-139, 2018.
32. Jiang J, Wang W, Sun YJ, Hu M, Li F and Zhu DY: Neuroprotective effect of curcumin on focal cerebral ischemic rats by preventing blood-brain barrier damage. Eur J Pharmacol 561: 54-62, 2007.

33. Wang C, Yang YH,Zhou L, Ding XL, Meng YC and Han K: Curcumin alleviates OGD/R-induced PC12 cell damage via repressing CCL3 and inactivating TLR4/MyD88/MAPK/NF- $\mathrm{KB}$ to suppress inflammation and apoptosis. J Pharm Pharmacol 72: 1176-1185, 2020.

34. Zong X, Wu S, Li F, Lv L, Han D, Zhao N, Yan X, Hu S and $\mathrm{Xu}$ T: Transplantation of VEGF-mediated bone marrow mesenchymal stem cells promotes functional improvement in a rat acute cerebral infarction model. Brain Res 1676: 9-18, 2017.

35. Yang $Y$ and Rosenberg GA: Blood-brain barrier breakdown in acute and chronic cerebrovascular disease. Stroke 42: 3323-3328, 2011.

36. Gerace E, Scartabelli T, Pellegrini-Giampietro DE and LanducciE:Toleranceinducedby(S)-3,5-dihydroxyphenylglycine postconditioning is mediated by the PI3K/Akt/GSK3 $\beta$ signalling pathway in an in vitro model of cerebral ischemia. Neuroscience 433: 221-229, 2020.

37. Eghbaliferiz S, Farhadi F, Barreto GE, Majeed M and Sahebkar A: Effects of curcumin on neurological diseases: Focus on astrocytes. Pharmacol Rep 72: 769-782, 2020.

38. Nery-Flores SD, Mendoza-Magaña ML, Ramírez-Herrera MA, Ramírez-Vázquez JJ, Romero-Prado MMJ, Cortez-Álvarez CR and Ramírez-Mendoza AA: Curcumin exerted neuroprotection against ozone-induced oxidative damage and decreased NF- $\kappa B$ activation in rat hippocampus and serum levels of inflammatory cytokines. Oxid Med Cell Longev 2018: 9620684, 2018.

39. Kodali M, Hattiangady B, Shetty GA, Bates A, Shuai B and Shetty AK: Curcumin treatment leads to better cognitive and mood function in a model of Gulf War Illness with enhanced neurogenesis, and alleviation of inflammation and mitochondrial dysfunction in the hippocampus. Brain Behav Immun 69: 499-514, 2018.

40. Lambertsen KL, Biber K and Finsen B: Inflammatory cytokines in experimental and human stroke. J Cereb Blood Flow Metab 32: 1677-1698, 2012.

41. Kar F, Hacioglu C, Senturk H, Donmez DB, Kanbak G and Uslu S: Curcumin and LOXblock-1 ameliorate ischemia-reperfusion induced inflammation and acute kidney injury by suppressing the semaphorin-plexin pathway. Life Sci 256: 118016, 2020.

42. Tang X, Sun L, Wang G, Chen B and Luo F: RUNX1: A regulator of NF-kB signaling in pulmonary diseases. Curr Protein Pept Sci 19: 172-178, 2017.

43. Zhu H, Dai R, Fu H and Meng Q: MMP-9 upregulation is attenuated by the monoclonal TLR2 antagonist T2.5 after oxygen-glucose deprivation and reoxygenation in rat brain microvascular endothelial cells. J Stroke Cerebrovasc Dis 28: 97-106, 2019.

44. Zhu H, Dai R, Zhou Y, Fu H and Meng Q: TLR2 ligand Pam3CSK4 regulates MMP-2/9 expression by MAPK/NF- $\kappa$ B signaling pathways in primary brain microvascular endothelial cells. Neurochem Res 43: 1897-1904, 2018.

45. Yang SL, Chen LJ, Kong Y, Xu D and Lou YJ: Sodium nitroprusside regulates mRNA expressions of LTC4 synthesis enzymes in hepatic ischemia/reperfusion injury rats via NF-kappaB signaling pathway. Pharmacology 80: 11-20, 2007.

46. Zhang S, An Q, Wang T, Gao S and Zhou G: Autophagyand MMP-2/9-mediated reduction and redistribution of ZO-1 contribute to hyperglycemia-increased blood-brain barrier permeability during early reperfusion in stroke. Neuroscience 377: 126-137, 2018.

47. Chang JJ, Emanuel BA, Mack WJ, Tsivgoulis G and Alexandrov AV: Matrix metalloproteinase-9: Dual role and temporal profile in intracerebral hemorrhage. J Stroke Cerebrovasc Dis 23: 2498-2505, 2014.

48. Liu WC, Wang X, Zhang X, Chen X and Jin X: Melatonin supplementation, a strategy to prevent neurological diseases through maintaining integrity of blood brain barrier in old people. Front Aging Neurosci 9: 165, 2017.

49. Xiong D, Deng Y, Huang B, Yin C, Liu B, Shi J and Gong Q: Icariin attenuates cerebral ischemia-reperfusion injury through inhibition of inflammatory response mediated by NF- $\mathrm{BB}$, PPAR $\alpha$ and PPAR $\gamma$ in rats. Int Immunopharmacol 30: 157-162, 2016.

50. Sun J, Guo W, Ben Y, Jiang J, Tan C, Xu Z, Wang X and Bai C: Preventive effects of curcumin and dexamethasone on lung transplantation-associated lung injury in rats. Crit Care Med 36: 1205-1213, 2008.

This work is licensed under a Creative Commons Attribution-NonCommercial-NoDerivatives 4.0 International (CC BY-NC-ND 4.0) License. 